\title{
Application of Uncertainty Modeling Frameworks to Uncertain Isosurface Extraction
}

\author{
Mahsa Mirzargar, Yanyan He, and Robert M. Kirby \\ Scientific Computing and Imaging Institute, University of Utah
}

\begin{abstract}
Proper characterization of uncertainty is a challenging task. Depending on the sources of uncertainty, various uncertainty modeling frameworks have been proposed and studied in the uncertainty quantification literature. This paper applies various uncertainty modeling frameworks, namely possibility theory, Dempster-Shafer theory and probability theory to isosurface extraction from uncertain scalar fields. It proposes an uncertainty-based marching cubes template as an abstraction of the conventional marching cubes algorithm with a flexible uncertainty measure. The applicability of the template is demonstrated using 2D simulation data in weather forecasting and computational fluid dynamics and a synthetic 3D dataset.
\end{abstract}

Keywords: Uncertainty quantification, isosurface visualization, possibility theory, Dempster-Shafer theory.

\section{Introduction}

Uncertainty is an inevitable component of predictive simulations based on computational models since the models are often imperfect or might involve uncertain parameters. As a result, understanding and quantifying the uncertainty in model output (data) is of critical importance.

To account for the uncertainty in data, an integral component of data analysis - visualization - has been combined with uncertainty modeling frameworks to constitute a special topic in the visualization community called uncertainty visualization. Uncertainty visualization is not a new topic and various uncertainty visualization techniques have been defined and studied [9, 13]. Most relevant one to the current work is isosurface extraction in the presence of uncertainty and hence, we focus only on visualization of uncertain isosurfaces. In order to quantify and visualize the uncertainty in isosurfaces extracted from uncertain scalar fields, parametric probabilistic models have been used to approximate Level-Crossing Probabilities (LCP) [15, 16]. The concept of levelcrossing probabilities has been deployed to extend the conventional marching cubes algorithm, the predominant isosurface visualization scheme, for probabilistic modeling of uncertainty in scalar fields [17, 18].

Probabilistic modeling is a well-developed approach for uncertainty when its source is a stochastic/random process (called aleatory uncertainty). However, it requires complete probability information for the random process, which could be quite difficult, if not impossible. Modeling epistemic uncertainty, referring to the uncertainty due to 
lack of knowledge [20], requires the exploration of the alternatives of probabilistic approaches. In the past few decades, possibility theory [6] and Dempster-Shafer (DS) theory [21], have been explored and studied in the uncertainty quantification literature for a better representation of the epistemic uncertainty. Yager [22] has developed a unified theoretical structure for uncertainty representation using various models.

In this work, we apply modern uncertainty modeling frameworks to the isosurface extraction from uncertain scalar fields, and propose an abstraction of the conventional marching cubes algorithm in terms of a template infrastructure that is flexible enough to incorporate various uncertainty modeling frameworks to model uncertainty in the spatial location of isosurfaces extracted from uncertain scalar fields. Our uncertaintybased marching cubes template can be considered as the extension of level-crossing probability to a general uncertainty measure.

The contributions of this work can be summarized as follows:

- We apply various uncertainty modeling frameworks to the isosurface extraction from uncertain scalar fields.

- We provide an abstraction of the marching cubes algorithm that we call uncertaintybased marching cubes (UMC) template. This template is amenable to deploy different mathematical frameworks for uncertainty modeling.

- We demonstrate the effectiveness and applicability of the template in a few examples of uncertain isosurface extraction from an ensemble of scalar fields.

The rest of the paper proceeds as follows. Section 2 is devoted to a brief introduction to the various uncertainty modeling frameworks. In Section 3 , we propose the uncertainty-based marching cubes template. Section 4 demonstrates the effectiveness of our template by presenting the results for several examples. We conclude our work in Section $\sqrt{5}$ and provide some avenues for future investigation.

\section{Mathematical Frameworks for Uncertainty Modeling}

Let $Y \in U \subseteq \mathbb{R}$ denote a random quantity of interest. We assume that the probability distribution of $Y$ is unknown, instead, we have a finite number of possible realizations of $Y$ (i.e., $\left\{y^{i}\right\}_{i=1}^{N}$ ) available. In such a situation, it is suggested that the random variable $Y$ should be represented "as a mixture of natural variability (aleatory) and estimation errors (epistemic)" since " a finite number of samples from a population leads to epistemic uncertainty [8]." The goal is to model the mixed uncertainty from the ensemble $\left\{y^{i}\right\}_{i=1}^{N}$ using uncertainty modeling frameworks. We consider the propositions in the form of "the true value of $Y$ is in $A$ " for any subset $A \subseteq U$, and adopt the measures from the uncertainty modeling frameworks to quantify the strength of the support from the evidence (i.e., the ensemble $\left\{y^{i}\right\}_{i=1}^{N}$ ) for proposition $A$. For example, the probability measure quantifies the chance of proposition $A$ being true.

In this section we introduce the mathematical notation used to express the various frameworks of fuzzy measures. Note: we skip the basics of probability theory due to the space limitations. Interested readers can consult [11]. 


\subsection{Possibility Theory}

Instead of a single measure in probability theory, possibility theory defines a dual measure (possibility and necessity) as [7]

$$
\operatorname{Poss}(A)=\max _{y \in A} \pi_{Y}(y), \quad \operatorname{Nec}(A)=1-\operatorname{Poss}\left(A^{c}\right),
$$

where $\pi_{Y}: U \rightarrow[0,1]$ is a possibility distribution, and $A^{c}$ is the complement of $A$. The possibility distribution describes the degree to which it is possible that the element $y \in U$ is the true value of $Y$.

The possibility function $\operatorname{Poss}(A)$ measures the maximum possible support from the evidence for proposition $A$ whereas the necessity function $N e c(A)$ quantifies the minimum support for proposition $A$. The length/distance between $N e c(A)$ and $\operatorname{Poss}(A)$ indicates the epistemic uncertainty regarding proposition $A$.

Assume that the $Y_{j}$ s are independent variables associated with possibility distribution $\pi_{Y_{j}}\left(y_{j}\right)$, the joint distribution is defined using $\min (\cdot, \cdot)$ as the joint operator [10 23]

$$
\pi_{\boldsymbol{Y}}(\boldsymbol{y})=\min \left(\min \left(\ldots \min \left(\pi_{Y_{1}}\left(y_{1}\right), \pi_{Y_{2}}\left(y_{2}\right)\right), \ldots\right), \pi_{Y_{M}}\left(y_{M}\right)\right) .
$$

Although there have been a few attempts to discover the correlation between uncertain variables in possibility theory [3], it is still an open problem to construct joint distribution for dependent variables from ensembles. This topic lies outside the scope of the current work, but remains an active area of research in the field of uncertainty quantification.

Construction of Possibility Distribution: Here, we provide two examples of constructing a possibility distribution.

1. With assumption of triangular shape distribution (referred as parametric technique): We construct a possibility distribution for the variable $Y$ based on a modified version of a triangular shape:

$$
\pi_{Y}(y)= \begin{cases}1-\frac{\left(y-y_{\text {mean }}\right)\left(1-p^{+}\right)}{y_{\max }-y_{\operatorname{mean}}} & \text { if } y_{\text {mean }} \leq y \leq y_{\max } \\ p^{+}+\frac{\left(y-y_{\min }\right)\left(1-p^{+}\right)}{y_{\text {mean }}-y_{\min }} & \text { if } y_{\text {min }} \leq y<y_{\text {mean }} \\ p^{+} & \text {otherwise }\end{cases}
$$

where $y_{\min }, y_{\text {mean }}$ and $y_{\max }$ are the minimum, sample mean and the maximum of the ensemble data, respectively; $p^{+}$is the upper bound of the probability of the true value of $Y$ falling outside the range of the ensemble data, which is estimated using Goodman's simultaneous confidence interval [4] as follows

$$
p^{+}=\frac{a+2 n+\sqrt{D}}{2(N+a)}, \quad D=a\left(a+\frac{4 n(N-n)}{N}\right),
$$

where $a$ is the quartile of order $1-\alpha$ of the chi-square distribution with one degree of freedom and $N$ is the size of the ensemble. There is no data that supports the value of $Y$ falling outside of the ensemble, and hence, $n=0$. The probability that the chance of "the value of $Y$ falls outside of the ensemble range" is less than $p^{+}$ is no less than $1-\alpha$, i.e., $\operatorname{Prob}\left(0 \leq \operatorname{Prob}\left(Y \notin\left[y_{\min }, y_{\max }\right]\right) \leq p^{+}\right) \geq 1-\alpha$ holds. Therefore, it is reasonable to assign $p^{+}$to the values outside the ensemble range in the possibility distribution. In the current work, we take one of the usual probability levels $\alpha=0.025$. 
2. Without assumption of shapes for distribution (referred as nonparametric technique): We construct a possibility distribution using the combination of histogram and the probability-possibility transformation proposed by Dubois et al. [5] as follows. The transformation Prob $\rightarrow \pi$ is based on the principle of maximum specificity, which aims at finding the most informative possibility distribution [5].

We first construct the probability distribution Prob from the histogram of the ensemble data $\left\{y^{i}\right\}_{i=1}^{N}$, where we fix the sample mean at the boundary of one of the bins of the histogram. Let $\left\{x_{j}\right\}_{j=1}^{l}$ be the bins and the probability values be $p_{j}=\operatorname{Prob}\left(x_{j}\right)$. If the probability values are ordered, i.e., $p_{1} \geq p_{2} \geq \ldots \geq p_{l}$, then a possibility distribution can be obtained using the transformation as follows:

$$
\pi_{1}=1, \quad \pi_{j}=\sum_{k=j}^{l} p_{k} .
$$

If there exist $j$ such that $p_{j}=p_{j+1}$, there will be different possibility distributions $\pi^{(t)}$ obtained for each permutation of the equal probability values. Then we choose the one that minimizes the possibility values as $\pi_{j}=\min _{t} \pi_{j}^{(t)}$. In order to provide smooth transition between the values in adjacent bins, we use a Gaussian filtering at the end for smoothing.

\subsection{Dempster-Shafer Theory}

Analogous to possibility theory, Dempster-Shafer (DS) theory also defines a dual measure (plausibility and belief), for $\forall A \subseteq U$, as

$$
P l(A)=\sum_{B \cap A \neq \emptyset} m(B), \quad B e l(A)=\sum_{B \subseteq A} m(B),
$$

where $m: 2^{U} \rightarrow[0,1]$ is a basic belief assignment (BBA), also called $m$-function. An $m$-function satisfies the following two conditions:

$$
m(\emptyset)=0, \quad \sum_{A \subseteq \mathbf{U}} m(A)=1 .
$$

The plausibility function $\operatorname{Pl}(A)$ and the belief function $\operatorname{Bel}(A)$ quantify the maximum and minimum strength of the evidence that supports the proposition, respectively. The length between $\operatorname{Bel}(A)$ and $P l(A)$ also indicates the epistemic uncertainty regarding proposition $A$.

Let $Y_{j}$ s be independent variables associated with basic belief assignments $m_{Y_{j}}\left(A_{j}\right)$ ( $A_{j} \in 2^{U_{j}}$ ), the joint basic belief assignment ( $m$-function) can be calculated by taking the product (joint operator) over all the components of $\boldsymbol{Y}=\left\{Y_{j}\right\}_{j=1}^{M}$ as [12]

$$
m(\boldsymbol{A})=m_{1}\left(A_{1}\right) m_{2}\left(A_{2}\right) \ldots m_{M}\left(A_{M}\right),
$$

where the hypercube $\boldsymbol{A}$ is the Cartesian product, i.e., $\boldsymbol{A}=A_{1} \times A_{2} \times \ldots \times A_{M}$.

Due to space limitations, we do not introduce further concepts for the dependent case, but refer the interested reader to [19].

Construction of Basic Belief Assignment: We construct belief/plausibility functions using the method proposed by Denœux [4] as follows. Consider two ordered consecutive intervals: $A_{1}=\{Y \leq \theta\}$ and $A_{2}=\{Y>\theta\}$ (the universal set becomes 
$\left.\left\{A_{1}, A_{2}\right\}\right)$, and "ordered" means that the elements in $A_{i}$ are no larger than the elements in $A_{j}$ if $i<j$. Let $n_{k}$ be the number of samples falling inside $\left\{A_{k}\right\}_{k=1}^{2}$ and

$$
P_{k}^{-}=\frac{a+2 n_{k}-\sqrt{D_{k}}}{2(N+a)}, \quad P_{k}^{+}=\frac{a+2 n_{k}+\sqrt{D_{k}}}{2(N+a)},
$$

where $a$ and $D_{k}$ are computed using the relation in Eq.4 4 with $n=n_{k}$. Let $A_{k, j}(k \leq j)$ denote the union $A_{k} \cup A_{k+1} \cup \ldots \cup A_{j}$. Then the $m$-function is constructed as

$$
\begin{aligned}
m\left(A_{k, j}\right)=P_{k}^{-}, \quad \text { if } j=k, & \\
m\left(A_{k, j}\right)=P^{-}\left(A_{k, j}\right)-P^{-}\left(A_{k+1, j}\right)-P^{-}\left(A_{k, j-1}\right), \quad \text { if } j=k+1, & \\
m\left(A_{k, j}\right)=P^{-}\left(A_{k, j}\right)-P^{-}\left(A_{k+1, j}\right)-P^{-}\left(A_{k, j-1}\right)+P^{-}\left(A_{k+1, j-1}\right), & \text { if } j>k+1,
\end{aligned}
$$

where

$$
P^{-}(B)=\max \left(\sum_{A_{k} \subset B} P_{k}^{-}, 1-\sum_{A_{k} \not \subset B} P_{k}^{+}\right), \quad \forall B \neq \emptyset .
$$

\section{Application of Uncertainty Modeling Frameworks to Isosurface Extraction}

In this section, we apply the introduced uncertainty modeling frameworks to isosurface extraction and introduce our uncertainty-based marching cubes (UMC) template. We first recall the fundamentals of the concept of level crossing in the (deterministic) marching cubes algorithm.

\subsection{Deterministic Marching Cubes Algorithm}

In the absence of uncertainty, a deterministic scalar field can be considered as a discrete representation of a continuous multivariate function $g(\cdot)$ using a set of deterministic scalar values $\left\{y_{j}\right\}_{j=1}^{M}$ on a grid, where $M$ denotes the resolution of the grid. An isosurface of $g(\cdot)$ associated with a given isovalue $\theta$ is defined as: $C=\left\{\boldsymbol{x} \in \mathbb{R}^{d}, g(\boldsymbol{x})=\theta\right\}$, where $d$ is the embedding dimension. The goal of the marching cubes algorithm (in $3 \mathrm{D})$ is to extract an approximation of the isosurface based on the trilinear approximation (i.e., tensor product of linear interpolation in the univariate case) of the underlying continuous function giving rise to the scalar field [14].

The local nature of the trilinear approximation simplifies the isosurface extraction significantly. Trilinear approximation requires the information only at the corners of a cell (e.g., a cube on a 3D Cartesian lattice). Therefore, the presence of a level crossing inside each cell (i.e., cell crossing) is locally determined based on the values of the scalar field at the corners of the cell. A cell crossing happens if at least one sign change occurs in the set of differences $\left\{y_{j}-\theta\right\}_{j=1}^{M}$ for the scalar values $y_{j}$ at the corners of the cell under question. In the presence of a cell crossing, the values at the corners of the cell also determine the (approximate) polygonal tessellation and the spatial location of the isosurface.

In the presence of uncertainty or error associated with the scalar field, the scalar values are no longer known deterministically. Consequently the spatial location of the isosurface from an uncertain scalar field becomes uncertain. Therefore, the conventional marching cubes algorithm must be extended to incorporate the uncertainty to provide reliable information about the presence or absence of an isosurface inside a cell. 


\subsection{Uncertain Cell Crossing and UMC Template}

We propose an uncertainty-based marching cubes (UMC) template that encompasses the essential concepts from the deterministic and probabilistic marching cubes algorithm [18] and is flexible enough to be adopted for various uncertainty modeling frameworks. Similar to the conventional marching cubes algorithm, the UMC template (algorithm) proceeds through the uncertain scalar field, and at each cell it quantifies how much the available information at the corners supports the incidence of a cell crossing.

To construct an abstract template, which accommodates various uncertainty modeling frameworks, we adopt a generic and flexible (uncertainty) measure that we call $U$-Measure to indicate the presence of cell crossing for each constituent cell of an uncertain scalar field (i.e., uncertain cell crossing). The U-Measure lends itself to various uncertainty modeling frameworks, and hence, the cell-crossing U-Measure value can be computed based on the axioms of a chosen uncertainty modeling framework.

For what follows, let us define a cell-crossing proposition:

$$
C: \quad \text { "there exists a level-crossing in a cell". }
$$

The goal is to evaluate the cell-crossing U-Measure values for proposition (12) (i.e., U-Measure $(C)$ ) for each cell in an uncertain scalar field. Computation of the U-Measure $(C)$ can be broken down into a few steps.

For every cell, the first step in computing U-Measure $(C)$ is to construct a joint distribution function based on the ensemble about the uncertain scalar values at the corners. As discussed in Section 2. construction of a joint distribution function can be car-

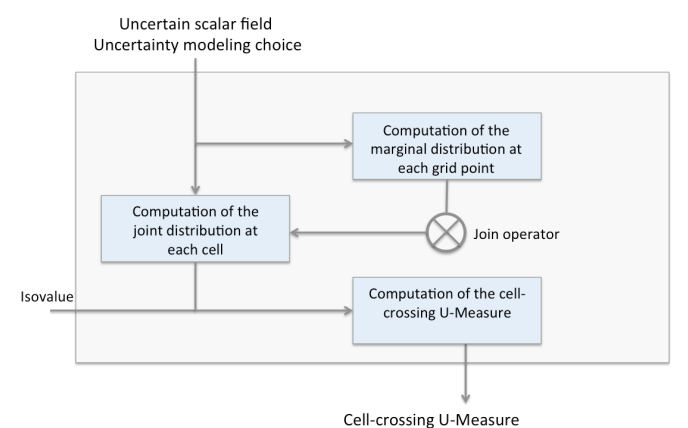

Fig. 1. Schematic illustration of the uncertainty-based marching cubes template. ried out either by

- first constructing a marginal distribution function at each grid point and then using a joint operator to define the joint distribution. For example, use Eqs. (3) or (5) in possibility theory, and Eqs. (10) and (8) in Dempster-Shafer theory.

- or in special cases, such as in the probabilistic case [18], directly from the available information at the corners.

The second step is to compute the cell-crossing U-Measure value(s) for proposition $C$ for a cell after construction of its joint distribution function. The value(s) of U-Measure $(C)$ for a cell can be evaluated using Eq. 1 for possibility theory or Eq. 6 for DS theory or Eq. (14) for probability theory. These steps constitute the building blocks of the uncertainty-based marching cubes template that has also been demonstrated schematically in Fig. 1 .

Without loss of generality, we illustrate the idea for a 1D example (similar to the example presented in [18]) while the concepts extend to higher dimensions. 
Consider two adjacent points as corners of an edge with associated (uncertain) scalar variables $y_{1}$ and $y_{2}$, respectively. Proposition $C$ is equivalent to considering the incidence of the values $\left(y_{1}, y_{2}\right)$ belonging to

$$
A=\left\{\left(y_{1}, y_{2}\right):\left(y_{1} \geq \theta \& y_{2} \leq \theta\right) \mid\left(y_{1} \leq \theta \& y_{2} \geq \theta\right)\right\}
$$

Now let us consider an ensemble of tuple values $\left(y_{1}, y_{2}\right)$ as the uncertain scalar values at the endpoints of an edge. A tuple from the ensemble results in an edge crossing if the linear interpolant connecting its endpoints crosses value $\theta$ (Fig. 2 (a)). Fig. 2 (b) illustrates another representation (scatterplot) of the set of (tuple) scalar values in Fig. 2 (a). Fig. 2 (b) makes it more clear that if the values of a tuple belong to one of the subregions marked as $A_{2}=\left\{y_{1} \geq \theta \& y_{2} \leq \theta\right\}$ or $A_{4}=\left\{y_{1} \leq \theta \& y_{2} \geq \theta\right\}$, the linear interpolant associated with them will result in an edge crossing. To evaluate the edge-crossing U-Measure values (i.e., the measure of the support for proposition $C$ from the available data), one needs to construct a joint distribution function (e.g., $f_{\boldsymbol{Y}}(\boldsymbol{y})$ in Fig. 2(c)) based on a chosen uncertainty modeling framework. The edge-crossing UMeasure values can then be computed as discussed earlier. For instance, in probabilistic modeling [18], probability measure is used to compute the U-Measure $(C)$ values as

$$
\text { U-Measure }(C)=\operatorname{Prob}(C)=\int_{A_{2} \cup A_{4}} f_{\boldsymbol{Y}}(\boldsymbol{y}), \quad \boldsymbol{y}=\left(y_{1}, y_{2}\right) \text {. }
$$

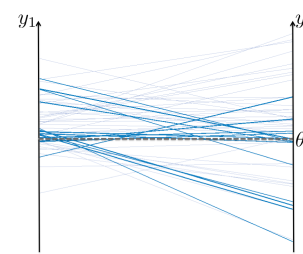

(a)

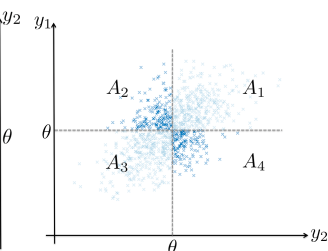

(b)

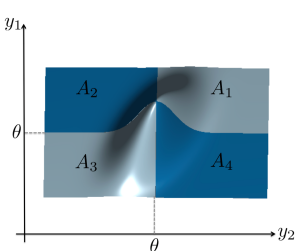

(c)

Fig. 2. (a) An ensemble of linear interpolants in 1D: the linear interpolants that correspond to the presence of an edge crossing are highlighted. (b) The values at the end points of each linear interpolant have been demonstrated as points in $\mathbb{R}^{2}$. The highlighted points correspond to tuples that result in presence of edge crossing in (a). (c) A joint distribution function $f_{\boldsymbol{Y}}(\boldsymbol{y})$ inferred by fitting a multivariate Gaussian function to the data in (b). The regions corresponding to the presence of edge crossing are highlighted in dark blue.

Note: i) The UMC template maintains a linear computational cost in terms of the number of the cells and takes advantage of the local nature of the concept of cell crossing similar to the deterministic version. However, it is important to note that the overall computational cost of instances (instantiations) of the UMC template for different uncertainty modeling frameworks is higher than the deterministic version due to the cost associated with the construction of the distribution function and computation of the UMeasure. ii) We focus only on the construction of the joint distributions that use an independent assumption (as examples) even though our proposed framework can easily incorporate dependence. 


\section{Results and Applications}

In this section, we demonstrate the utility of the UMC template with three experimental examples from each uncertainty modeling framework. In all the examples, the colormap has been scaled so that the highest level-crossing U-Measure value is assigned to blue and the minimum level-crossing U-Measure value is assigned to white. The scaling of the colormap helps to provide better color contrast.

Note: The goal of the current work is to demonstrate the applicability of the UMC template with different mathematical uncertainty modeling frameworks. The comparisons among the mathematical modeling frameworks and among the corresponding isocontour/isosurface extraction results are beyond the scope of the current manuscript; they represent present and future work within the uncertainty quantification field which may in part facilitated by our template. Therefore, we present each example with specific mathematical modeling framework and the corresponding visualization results.

\subsection{Temperature Forecast Example Using Possibility Theory}

We demonstrate the UMC template with possibility theory using a dataset from the weather forecast application. For this example, we use one of the publicly available weather forecast ensembles called SREF-CONUS $(40 \mathrm{~km})$ temperature ensemble by NOAA [1]. This ensemble consists of 21 members that are generated by varying the forecast model and the initial conditions to account for various sources of uncertainty (both model and parameter uncertainty). We have chosen to use one of the predefined temperature isovalues adopted by NOAA that is $-15 \mathrm{C}$ at $500 \mathrm{mb}$.

For this example, we use both parametric (Eq. 3) and nonparametric (Eq. 5 ) techniques to construct possibility distributions. First two rows of Fig. 3 provides the visualization of the level-crossing possibility and necessity values in both parametric and nonparametric settings. The possibility values suggest the maximum/optimistic estimation for the chance of the presence of level-crossing at each cell, and the region near the mean isocontour (visualized in black) has a relatively higher maximum chance of the presence of level crossing. The necessity values indicate the minimum/conservative estimation of the chance of the presence of level crossing. Note that the colormap for necessity values has been flipped to make them more visible. The region with parametric nonzero necessity values is coincident with the mean isocontour, which is due to (a) the degree of possibility $\pi(y)=1$ is assigned to the mean ensemble $y_{\text {mean }}$ when we construct the possibility distribution; and (2) the relation $N e c(A) \neq 0$ if $\operatorname{Poss}(A)=1$.

Note that the lack of smoothness of the computed possibility/necessity values in the nonparametric case is due to the oscillatory nature of the possibility distribution function in the nonparametric setting. Instances of the distribution functions constructed from an ensemble at one grid location are shown in Fig. 3 (c) and Fig. 3 (f). The parametric technique of constructing possibility distribution has fewer degrees of freedom compared to the nonparametric technique; therefore the resulting distribution function from the parametric technique is less oscillatory.

\subsection{Computational Fluid Dynamics Example Using DS Theory}

We use DS theory as the underlying mathematical framework for instantiation of the uncertainty-based marching cubes template, and demonstrate the results for a fluid sim- 


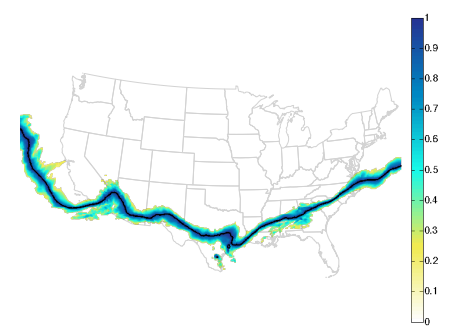

(a) parametric cell-crossing possibility

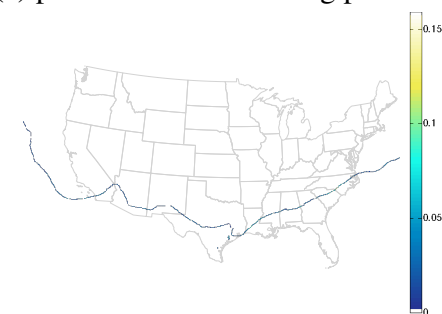

(b) parametric cell-crossing necessity

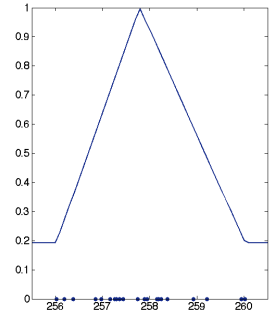

(c) parametric distribution function

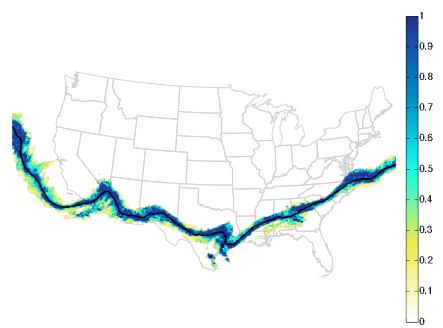

d) nonparametric cell-crossing possibility

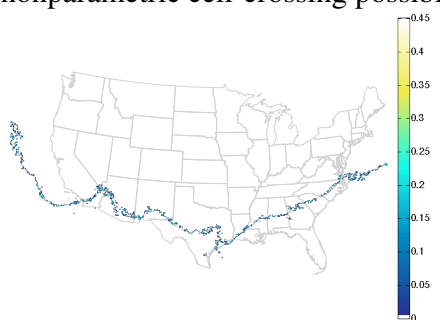

(e) nonparametric cell-crossing necessity

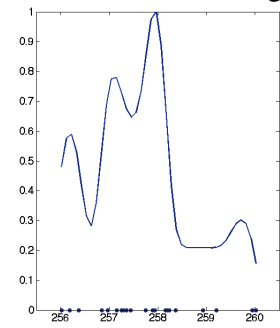

(f) nonparametric distribution function

Fig. 3. The UMC template instantiated with possibility theory. Figures (a-c) demonstrate the results for parametric and figures (d-f) demonstrate the results for nonparametric technique. The possibility distribution demonstrated in (c) and (f) are constructed from an ensemble at a single point in the dataset (the ensemble values are represented with dots).

ulation example, which is motivated by the use of ensembles in computational fluid dynamics to study structures such as vortices.

For this example, we use the simulation of flow past a circular cylinder. When the fluid passes an obstacle, the eddies or vortices are formed in a periodic fashion and move in the direction of the flow field shown in Fig. 4 (a). Studying the pressure of a flow field is among the simplest approaches to study vortex structures. The center of a vortex typically corresponds to minimum pressure values. Therefore, isocontours of the pressure field can be used to approximate the position and size of the vortices in a flow field. The number and the position of vortices generated is affected by variation of the simulation parameters such as the Reynolds number, initial conditions and boundary conditions.

For this example, we used the 2D incompressible Navier-Stokes solver as part of 


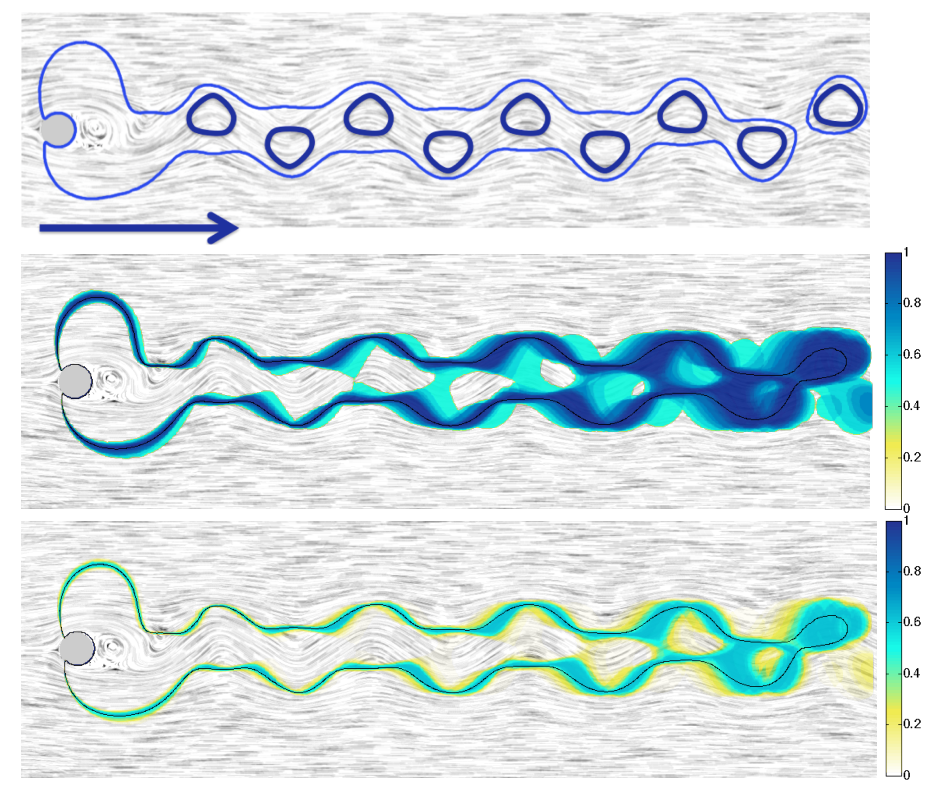

Fig. 4. (top) An example of the vortex street formed by a flow past a cylinder, (middle) cellcrossing plausibility values, (bottom) cell-crossing belief values.

the Nektar++ software package [2] to generate simulation results for fluid passing our stationary obstacle. We generated our ensemble of size 40 by random perturbation of the inlet velocity and the Reynolds number. After normalizing the pressure field of each ensemble member based on the average of the pressure value for a unique and fixed point inside

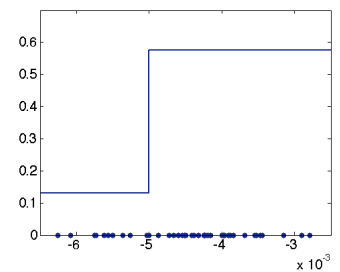

Fig. 5. An $m$-function constructed from an ensemble (dots). the field behind the cylinder, we have chosen isovalue $=-0.005$.

We constructed the basic belief assignment ( $m$-function) using the technique discussed in Section 2. The cell-crossing belief and plausibility values (i.e., U-Measure in DS theory) are visualized in Fig. 4. The plausibility values indicate the maximum chance of level crossing at each cell whereas the belief values indicate the minimum chance. As an example, the $m$-function constructed from the ensemble at one grid location is shown in Fig. 5 and is constructed as

$$
m(\{y<=\theta\})=0.1306, m(\{y>\theta\})=0.5748,
$$

and $m\left(A_{1} \cup A_{2}\right)=1-m\left(A_{1}\right)-m\left(A_{2}\right)$ where $A_{1}=\{y<=\theta\}, A_{2}=\{y>\theta\}$. As Fig. 5 demonstrates, the constructed $m$-function is not oscillatory and hence the resultant U-Measures values have smooth transitions.

\subsection{Synthetic Example Using Probability Theory}

In the last example, we show that our UMC template is applicable to a high-dimensional dataset. Specifically, we provide the result of our UMC template on a 3D synthetic 
example given in [18] through volume rendering of the probability cell-crossing values (i.e., the U-Measure in probability theory). The synthetic data contains an ensemble of 45 volumetric data using the analytical formula: $\mu(x, y, z)=(\cos (7 x)+\cos (7 y)+$ $\cos (7 z) \exp (a r)$ where $r=\sqrt{x^{2}+y^{2}+z^{2}}$ and $a=-4.5$. Each ensemble member is a volumetric data of size $300 \times 300 \times$ 250 where $a$ has been perturbed by adding normally distributed noise values. For this example, we use our UMC template with the probabilitytheory-based parametric modeling assumption. In this case, the instantiation of our template is equivalent to the probabilistic marching cubes algorithm [18] and the U-Measure in this case is the chance of the presence of level crossing (i.e., probability values). Fig. 6 shows that probability values are much smaller

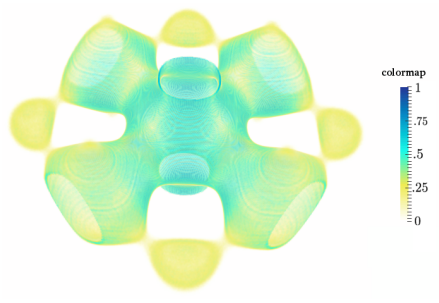

Fig. 6. 3D Uncertainty-based marching cubes: with a parametric modeling assumption, the UMC (template) translates into probabilistic marching cubes [18].

around the corners of the volumetric data compared to the values at the center.

\section{Summary and Conclusions}

This paper applies possibility theory and Dempster-Shafer (DS) theory as alternative uncertainty modeling frameworks of probability theory to the isosurface extraction from uncertain scalar fields. It proposes an uncertainty-based marching cubes template as an abstraction of the conventional marching cubes algorithm with a flexible uncertainty measure. The effectiveness and applicability of the template are demonstrated in a few examples of uncertain isosurface extraction from an ensemble of scalar fields.

In the future, the proposed framework can be used to further study the choice of different mathematical frameworks for the representation and quantification of specific types of uncertainty (aleatoric, epistemic or their mixture) in different data formats. Some of the limitations of the current work also suggest interesting avenues for future research. For example, designing techniques to infer dependence structure suitable for possibility and Dempster-Shafer theories is an interesting avenue of future research that can potentially result in improving the quality and accuracy of uncertainty modeling using these theories; and decision making based on visualization of the results of dual measures could be an interesting future research direction as well.

Acknowledgement: The first and third authors acknowledge the support of National Science Foundation (NSF) grant IIS-1212806. The second author acknowledges support by the Army Research Laboratory under Cooperative Agreement Number W911 NF-12-2-0023. The views and conclusions contained in this document are those of the authors and should not be interpreted as representing the officialpolicies, either expressed or implied, of the Army Research Laboratory or the U.S. Government. The U.S. Government is authorized to reproduce and distribute reprints for Government purposes notwithstanding any copyright notation herein. 


\section{References}

1. National oceanic and atmospheric administration (2015), http://www.noaa.gov/

2. Nektar++ (2015), http://www.nektar.info

3. Carlsson, C., Fullér, R., Majlender, P.: On possibilistic correlation. Fuzzy Sets and Systems 155(3), 425-445 (2005)

4. Denœux, T.: Constructing belief function from sample data using multinomial confidence regions. International Journal of Approximate Reasoning 42, 228-252 (2006)

5. Dubois, D., Foulloy, L., Mauris, G., Prade, H.: Probability-possibility transformations, triangular fuzzy sets, and probabilistic inequalities. Reliable Computing 10, 273-297 (2004)

6. Dubois, D., Prade, H.: Possibility theory. Plenum Press, New-York (1988)

7. Dubois, D.: Possibility theory and statistical reasoning. Computational Statistics \& Data Analysis 51(1), 47-69 (2006)

8. Iaccarino, G.: Uncertainty quantification in computational science (2011), http://web. stanford.edu/group/uq/events/pdfs/lecture_2.pdf

9. Johnson, C., Sanderson, A.: A next step: Visualizing errors and uncertainty. IEEE Computer Graphics and Applications 23 (2003)

10. Klir, G., Yuan, B.: Fuzzy Sets and Fuzzy Logic: Theory and Applications. Prentice-Hall Inc. (1995)

11. Kolmogorov, A.: Foundations of the Theory of Probability. Chelsea Pub Co (Jun 1960)

12. Kriegler, E., Held, H.: Utilizing belief functions for the estimation of future climate change. International Journal of Approximate Reasoning 39, 185-209 (2005)

13. Lodha, S., Wilson, C., Sheehan, R.: Listen: sounding uncertainty visualization. In: Proceedings of IEEE Visualization. pp. 189-195 (1996)

14. Lorensen, W.E., Cline, H.E.: Marching cubes: A high resolution 3d surface construction algorithm. COMPUTER GRAPHICS 21(4), 163-169 (1987)

15. Pfaffelmoser, T., Reitinger, M., Westermann, R.: Visualizing the positional and geometrical variability of isosurfaces in uncertain scalar fields. Computer Graphics Forum 30(3), 951$960(2011)$

16. Pöthkow, K., Hege, H.C.: Positional uncertainty of isocontours: Condition analysis and probabilistic measures. IEEE Trans. on Visualization and Computer Graphics 17(10), 1393-1406 (2011)

17. Pöthkow, K., Petz, C., Hege, H.C.: Approximate level-crossing probabilities for interactive visualization of uncertain isocontours. International Journal for Uncertainty Quantification 3(2), 101-117 (2013)

18. Pöthkow, K., Weber, B., Hege, H.C.: Probabilistic marching cubes. Computer Graphics Forum 30(3), 931-940 (2011)

19. Regan, H., Ferson, S., Berlent, D.: Equivalence of methods for uncertainty propagation of real-valued random variables. International Journal of Approximate Reasoning 36, 1-30 (2004)

20. Roy, C.J., Oberkampf, W.L.: A comprehensive framework for verification, validation, and uncertainty quantification in scientific computing. Computer Methods in Applied Mechanics and Engineering 200, 2131 - 2144 (2011)

21. Shafer, G.: A Mathematical Theory of Evidence. Princeton University Press (1976)

22. Yager, R.R.: A general approach to uncertainty representation using fuzzy measures. In: FLAIRS Conference. pp. 619-623. AAAI Press (2001)

23. Zadeh, L.A.: Fuzzy sets as a basis for a theory of possibility. Fuzzy Sets and Systems (1), 3-28 (1978) 